\title{
On Robust Signal Reconstruction in Noisy Filter Banks
}

\author{
Haris Vikalo ${ }^{\dagger}$, Babak Hassibi ${ }^{\ddagger}$, Alper Erdogan*, and Thomas Kailath ${ }^{\dagger}$ \\ ${ }^{\dagger}$ Information Systems Lab, Stanford University, Stanford CA 94309, USA \\ ${ }^{\ddagger}$ Department of Electrical Engineering, Caltech, Pasadena CA 91106, USA \\ ${ }^{*}$ Department of Electrical Engineering, Koc University, Istanbul, Turkey
}

\begin{abstract}
We study the design of synthesis filters in noisy filter bank systems using an $H^{\infty}$ estimation point of view. The $H^{\infty}$ approach is most promising in situations where the statistical properties of the disturbances (arising from quantization, compression, etc.) in each subband of the filter bank is unknown, or is too difficult to model and analyze. For the important special case of unitary analysis polyphase matrices we obtain an explicit expression for the minimum achievable disturbance attenuation. For arbitrary analysis polyphase matrices, standard state-space $H^{\infty}$ techniques can be employed to obtain numerical solutions. When the synthesis filters are restricted to being FIR, as is often the case in practice, the design can be cast as a finite-dimensional semi-definite program. In this case, we can effectively exploit the inherent non-uniqueness of the $H^{\infty}$ solution to optimize for an additional criteria. By optimizing for average performance in addition to the $H^{\infty}$ criteria, we obtain mixed $H^{2} / H^{\infty}$ optimal FIR synthesis filters. Alternatively, if the additional criteria is concerned with penalizing occasional occurance of large values of reconstraction errors more than frequent occurance of small to moderate ones, we obtain risksensitive FIR synthesis filters. Numerical examples and comparisons with existing methods are also included.
\end{abstract}

Key words:

$P A C S$ : 


\section{Introduction}

Multirate filter bank systems have been a subject of extensive studies (see [1][3] and the references therein) and are widely used in many application areas (such as speech and image compression, joint source channel coding, adaptive systems, and others). The design of perfect reconstruction filter banks, capable of exactly replicating the input signal, has received particularly high attention. In most of the research, the subbands of the filter bank system are assumed noise free. Such an $M$-band filter bank system is illustrated in Figure 1. The analysis filters $H_{i}(z)$ decompose the input signal into subband components, which are then decimated by a factor of $M$. The signal is reconstructed by upsampling by a factor of $M$ followed by filtering with synthesis filters $F_{i}(z)$.

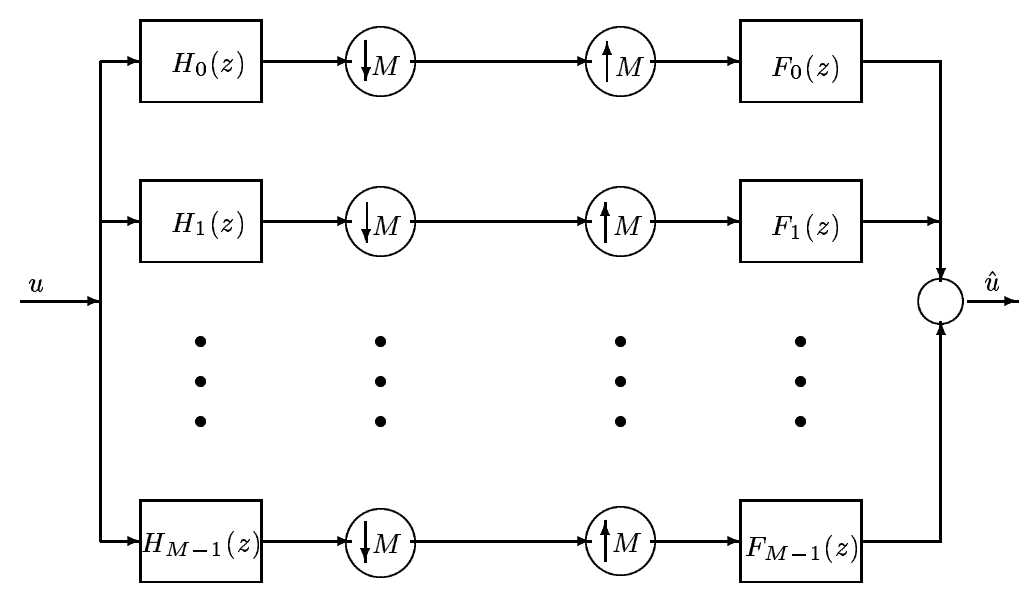

Fig. 1. M-channel filter bank

Ideally, the synthesis filters are required to exactly reconstruct the delayed version of the input signal. The perfect reconstruction is thus achieved if

$$
\hat{u}_{i}=u_{i-m}
$$

where $m>0$ is the delay. However, the decimated signals in the subbands may be, for example, encoded and transmitted (as in speech comparison applications), or be coded for storage, at which point the signal may be compressed and some information lost. The perfect reconstruction approach studied in the literature, assumes no loss of information in the subbands. However, signal quantization and noise corruption in the subbands, as well as computational roundoff, are always present in practical filter banks systems [4],[5]. Thus, noise in subbands must be carefully considered in systems design.

In order to deal with noise-corrupted filter bank systems, multirate Kalman synthesis filtering has been recently proposed [6]. The Kalman filtering approaches require a priori knowledge of the (first and second-order) noise statis- 
tics. Therefore in applications involving compression, quantization, etc., where the noise statistics are not readily known, the performance of the synthesis filters may be suspect.

$H^{\infty}$ estimation, on the other hand, requires no statistical assumptions, performs a worst-case design, and is therefore robust with respect to noise uncertainty. Moreover, the $H^{\infty}$ approach allows one to explicitly introduce finite delay into the synthesis filter design. [Conventional IIR perfect reconstruction filters are often non-causal, which requires either infinite delay or some form of truncation.] The $H^{\infty}$ optimization approach had been proposed first in [7], followed by [8]-[10]. Unlike this paper, the work in [7]-[9] considered only the noise-free subband case (in other words, we are considering a two-block estimation problem, rather than the one-block problem considered in [7]-[9]).

The existence of corrupting noises in the subbands of the filter bank systems is assumed throughout this paper. Analysis filters can be designed for good frequency selectivity (i.e., good coding of the input signal). Then the synthesis filters are designed to minimize the maximum energy gain from the unknown disturbances to the estimation errors, i.e., the goal is to minimize the worstcase reconstruction error to disturbance ratio.

We take on state space approach (unlike, say, [11]) to the design of synthesis filters. In particular, we pose the design problem in terms of matrices in the state space model of the polyphase representation of the synthesis filters. Furthermore, by assuming FIR analysis filters, we state the optimization problem explicitely in terms of the coefficients of the synthesis filters. This differs from the work in [10], where the state space representation of the bank of filters is used to find an appropriate estimation gain. Finally, the results on closed-form expression for the $H^{\infty}$ optimal synthesis filters for paraunitary filter banks and derivation of the risk sensitive synthesis filters have not been obtained elsewhere.

The outline of the paper is as follows. The model description is given in Section 2, where the problem is made equivalent to a standard general estimation problem. In Section 3, we review the $H^{\infty}$ approach to the estimation problem, apply it to the design of synthesis filters in the filter bank, and derive closedform $H^{\infty}$ optimal solution for the special case of paraunitary analysis filters. In Section 4, assuming FIR analysis filters, we give a state-space solution to the problem via semi-definite programming. Optimal mixed $H^{2} / H^{\infty}$ design is discussed in Section 5. In Section 6, we give simulation results and compare the approach taken here with existing methods. Conclusions are given in Section 7 . 


\section{Model Description}

To begin our study, we will use a polyphase representation of the filter bank shown in Figure 1. We can represent the analysis filter bank in terms of the $M \times M$ polyphase matrix $H(z)$

$$
H(z)=\left[\begin{array}{cccc}
H_{0,0}(z) & H_{0,1}(z) & \cdots & H_{0, M-1}(z) \\
H_{1,0}(z) & H_{1,1}(z) & \cdots & H_{1, M-1}(z) \\
\vdots & \vdots & \ddots & \vdots \\
H_{M-1,0}(z) & H_{M-1,1}(z) & \cdots & H_{M-1, M-1}(z)
\end{array}\right]
$$

where $H_{k, l}$ is the $l$ th polyphase component of the $k$ th analysis filter. One can find the polyphase analysis matrix $H(z)$ by performing a type-1 polyphase decomposition of the analysis filters as in [1],

$$
\left[\begin{array}{c}
H_{0}(z) \\
H_{1}(z) \\
\vdots \\
H_{M-1}(z)
\end{array}\right]=H\left(z^{M}\right)\left[\begin{array}{c}
1 \\
z^{-1} \\
\vdots \\
z^{-(M-1)}
\end{array}\right]
$$

For the synthesis filter bank, we can define a polyphase matrix $F(z)$ in the similar manner,

$$
F(z)=\left[\begin{array}{cccc}
F_{0, M-1}(z) & F_{0, M-2}(z) & \cdots & F_{0,0}(z) \\
F_{1, M-1}(z) & F_{1, M-2}(z) & \cdots & F_{1,0}(z) \\
\vdots & \vdots & \ddots & \vdots \\
F_{M-1, M-1}(z) & F_{M-1, M-2}(z) & \cdots & F_{M-1,0}(z)
\end{array}\right]
$$

and find it by performing a type- 2 polyphase decomposition of the synthesis filters,

$$
\left[\begin{array}{llll}
F_{0}(z) & F_{1}(z) & \cdots & F_{M-1}(z)
\end{array}\right]=\left[\begin{array}{llll}
z^{M-1} & \cdots & z & 1
\end{array}\right] F\left(z^{M}\right) .
$$

Thus by replacing the bank of filters by its polyphase equivalent, we obtain the polyphase equivalent structure of Figure 2. The subband noises are considered to be additive noises in this model. 


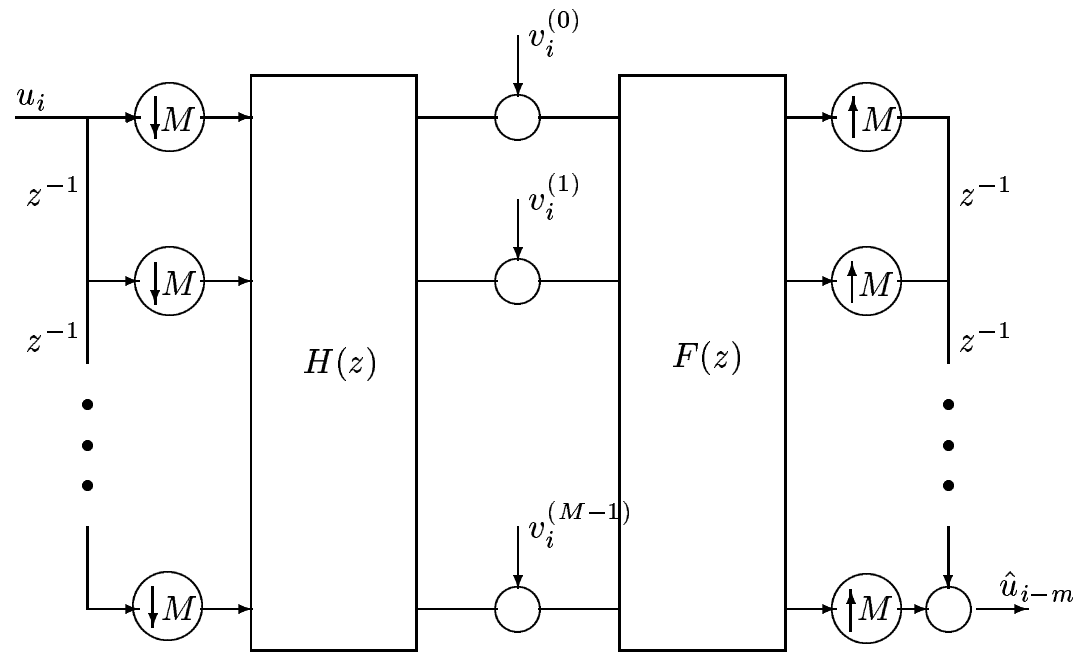

Fig. 2. Polyphase equivalent structure

Blocking the input and output (see [7]) leads to a so-called vector-matrix equivalent structure in Figure 3. The input signal $\mathbf{u}_{i}$ (bold symbol denotes a vector) in Figure 3 is of the form

$$
\mathbf{u}_{i}=\left[\begin{array}{llll}
u_{i M} & u_{i M-1} & \ldots & u_{i M-M+1}
\end{array}\right]^{T} .
$$

Notice that while the original system in Figure 1 is a linear periodic timevarying, the obtained equivalent system in Figure 3 is linear time-invariant. As noted before, we are interested in estimating $u_{i-m}$, the delayed version of the input signal $(m>0)$. The transfer matrix $L(z)$ in Figure 3 can be found as

$$
\begin{gathered}
L(z)=z^{-d}\left[\begin{array}{cc}
0_{(M-k) \times k} & I_{(M-k) \times(M-k)} \\
z^{-1} I_{k \times k} & 0_{k \times(M-k)}
\end{array}\right], \\
m=M d+k, k=0,1, \ldots, M-1 .
\end{gathered}
$$

Notice the paraunitary property of $L(z)$, i.e., that $L(z) L^{*}\left(z^{-*}\right)=L^{*}\left(z^{-*}\right) L(z)=$ $I$.

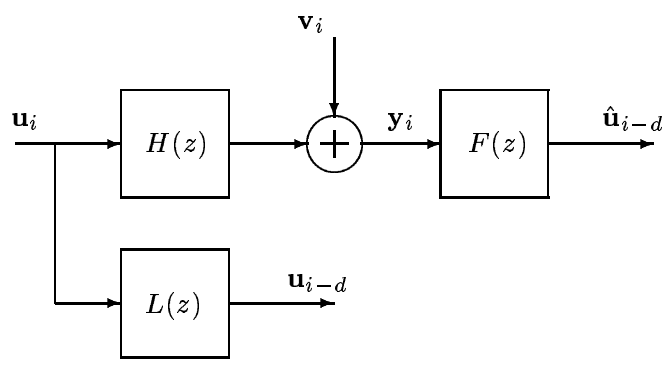

Fig. 3. Vector-matrix equivalent structure 
The system in Figure 3 is the standard model for a general estimation problem, where the goal is to design the causal linear time-invariant estimator $F(z)$ to estimate the input sequence $\left\{\mathbf{u}_{i-d}\right\}$ from the observations $\left\{\mathbf{y}_{i}\right\}$. The performance of the estimator is evaluated according to an adopted criterion. One common choice for such a criterion is the expected squared estimation error, $E\left(\mathbf{u}_{i}-\hat{\mathbf{u}}_{i}\right)^{*}\left(\mathbf{u}_{i}-\hat{\mathbf{u}}_{i}\right)$, which is minimized by a minimum-mean-square-error (MMSE) estimator. MMSE estimators assume that the disturbance sequences are zero-mean stationary processes with known second-order statistics. However, in practice, the first and second order statistics of the underlying disturbance are often not known exactly. One possible remedy for such a case is the design of an estimator that would guarantee that small disturbances lead to small estimation errors regardless of the statistical properties of the disturbances. This is, in fact, the defining property of $H^{\infty}$ estimators. An obvious drawback is that since no assumption about the disturbances is made, $H^{\infty}$ estimators may be over-conservative.

\subsection{Paraunitary Filter Bank}

For arbitrary analysis filters, one can solve the equivalent $H^{\infty}$ estimation problem and find the corresponding synthesis filters using a variety of methods such as solving Riccati equations, performing $J$-spectral factorization, etc. (see, e.g., $[13,17,24]$ and the references therein). However, we should mention that the filter banks studied most extensively in the literature (especially in regards to perfect reconstruction solutions) are orthogonal filter banks (see, e.g., [1]). Such filter banks lead to paraunitary (or scaled paraunitary) polyphase anal-

ysis matrices. In the next section we shall specifically consider the design and performance of such filter banks from the $H^{\infty}$ estimation point of view. For this particular choice of analysis filters, we shall derive a closed form expression for the performance of the optimal synthesis filters in terms of our adopted criterion for the reconstruction error.

\section{$3 \quad H^{\infty}$ Approach}

With the polyphase representation of the filter bank, the design of the synthesis polyphase matrix can be regarded as a special case of the estimation problem formulation, where the observations $\left\{\mathbf{y}_{i}\right\}$ are the noise corrupted subbands signals. The induced transfer matrix mapping the unknown disturbances $\mathbf{u}_{i}$ and $\sigma^{-1} \mathbf{v}_{i}$ to the estimation errors is

$$
T_{F}(z)=[L(z)-F(z) H(z)-\sigma F(z)]
$$


where $\sigma^{2}$ represents the intensity of the noise. The goal of $H^{\infty}$ estimation is to choose a causal $F(z)$ to minimize the $H^{\infty}$ norm of $T_{F}(z)$.

Definition 1 (The $H^{\infty}$ Norm) Let the transfer matrix $T_{F}(z)$ map the input sequence $\left\{\mathbf{u}_{i}\right\}$ to the output sequence $\left\{\mathbf{y}_{i}\right\}$. Then the $H^{\infty}$ norm of $T_{F}(z)$ is defined as

$$
\left\|T_{F}(z)\right\|_{\infty} \triangleq \sup _{\mathbf{u} \in l^{2}, \mathbf{u} \neq \mathbf{0}} \frac{\|\mathbf{y}\|_{2}}{\|\mathbf{u}\|_{2}}
$$

where $\|\mathbf{u}\|_{2}^{2} \triangleq \sum_{i=-\infty}^{\infty} \mathbf{u}_{i}^{*} \mathbf{u}_{i}$ and $l^{2}$ denotes the Hilbert space of all squaresummable discrete-time signals.

In other words, the $H^{\infty}$ norm of a stable LTI system is the square-root of its maximum energy gain (more precisely, its $l^{2}$-induced norm). Applied to a filter bank system, we can state the design problem as follows:

Problem 1 (Optimal $H^{\infty}$ filter bank design) Given a polyphase analysis matrix $H(z)$, and a delay $d>0$, find a causal polyphase synthesis matrix $F(z)$ that solves for

$$
\inf _{\text {causal }(\cdot)} \frac{\sum_{i}\left(\mathbf{u}_{i-d}-\hat{\mathbf{u}}_{i-d}\right)^{*}\left(\mathbf{u}_{i-d}-\hat{\mathbf{u}}_{i-d}\right)}{\sum_{i} \mathbf{u}_{i}^{*} \mathbf{u}_{i}+\sigma^{-2} \sum_{i} \mathbf{v}_{i}^{*} \mathbf{v}_{i}} \triangleq \gamma_{o p t}^{2} .
$$

and find the resulting $\gamma_{o} p t$.

We should further remark that the frequency domain characterization of the $H^{\infty}$ norm is given by

$$
\left\|T_{F}(z)\right\|_{\infty}=\sup _{0 \leq \omega \leq 2 \pi} \bar{\sigma}\left[T_{F}\left(e^{j \omega}\right)\right]=\sup _{0 \leq \omega \leq 2 \pi} \lambda_{m a x}^{1 / 2}\left[T_{F}\left(e^{j \omega}\right) T_{F}^{*}\left(e^{j \omega}\right)\right]
$$

where $\bar{\sigma}(\cdot)$ denotes the maximum singular value of its argument.

There is also a related noncausal $H^{\infty}$ estimation problem, for which is very easy to obtain the closed-form solution.

Theorem 1 (Noncausal $H^{\infty}$-optimal Estimator) The optimal $H^{\infty}$ norm for the problem

$$
\inf _{F(\cdot)} \frac{\sum_{i}\left(\mathbf{u}_{i}-\hat{\mathbf{u}}_{i}\right)^{*}\left(\mathbf{u}_{i}-\hat{\mathbf{u}}_{i}\right)}{\sum_{i} \mathbf{u}_{i}^{*} \mathbf{u}_{i}+\sigma^{-2} \sum_{i} \mathbf{v}_{i}^{*} \mathbf{v}_{i}} \triangleq \gamma_{o p t}^{2}
$$


is given by

$$
\gamma_{s}=\left\|L(z)\left[I+\sigma^{-2} H^{*}\left(z^{-*}\right) H(z)\right]^{-1} L^{*}\left(z^{-*}\right)\right\|_{\infty}^{1 / 2}
$$

while one corresponding noncausal $H^{\infty}$-optimal solution is given by the Wiener smoother,

$$
F_{s}(z)=L(z) H^{*}\left(z^{-*}\right)\left[\sigma^{2} I+H(z) H^{*}\left(z^{-*}\right)\right]^{-1}
$$

The proof can be found in, e.g., [13]. Note, clearly, that $\gamma_{s} \leq \gamma_{\text {opt }}$ since noncausal solutions should outperform causal ones.

The optimal $H^{\infty}$ design with a causality constraint in place is hard to solve and, unlike the noncausal case that we just saw, closed-form expression for the causal $H^{\infty}$-optimal estimators are generally not available. The common approach is then to relax the optimization condition and solve for a related suboptimal estimation problem. We can state it in the filter bank context as follows:

Problem 2 (Suboptimal $H^{\infty}$ filter bank design) Given a $\gamma>0$, find $a$ causal synthesis polyphase matrix $F(z)$ that guarantees

$$
\left\|T_{F}(z)\right\|_{\infty}=\|[L(z)-F(z) H(z) \quad-\sigma F(z)]\|_{\infty}<\gamma
$$

We notice that it must hold that $\gamma \geq \gamma_{o p t}$.

For the sake of further discussion and for the completeness of this overview, we now state the solution to the suboptimal $H^{\infty}$ estimation problem. The solution, as we shall state in the following theorem, is based upon a procedure referred to as J-spectral factorization. In this paper, we shall not dwell on Jspectral factorization as a tool for solving $H^{\infty}$ optimization problems. Instead, for implementation purposes, we refer the reader to [7] for useful pointers to the relevant MATLAB tools. The purpose of the following theorem is to recall the non-unique character of the solution to the $H^{\infty}$ optimization problem.

Theorem 2 ( $H^{\infty}$ Suboptimal Filter Bank Design) Given $\gamma>0$, a causal $F(z)$ achieving

$$
\|[L(z)-F(z) H(z) \quad-\sigma F(z)]\|_{\infty}<\gamma
$$


exists if, and only if, there exists a so-called $J$-spectral factorization of the form

$$
\begin{aligned}
& {\left[\begin{array}{cc}
I+H(z) H^{*}\left(z^{-*}\right) & -H(z) L^{*}\left(z^{-*}\right) \\
-L(z) H^{*}\left(z^{-*}\right) & -\gamma^{2} I+L(z) L^{*}\left(z^{-*}\right)
\end{array}\right]=} \\
& {\left[\begin{array}{cc}
L_{11}(z) & L_{12}(z) \\
L_{21}(z) & L_{22}(z)
\end{array}\right]\left[\begin{array}{cc}
I & 0 \\
0 & -I
\end{array}\right]\left[\begin{array}{c}
L_{11}^{*}\left(z^{-*}\right) L_{12}^{*}\left(z^{-*}\right) \\
L_{21}^{*}\left(z^{-*}\right) L_{22}^{*}\left(z^{-*}\right)
\end{array}\right],}
\end{aligned}
$$

where $\left[\begin{array}{ll}L_{11}(z) & L_{12}(z) \\ L_{21}(z) & L_{22}(z)\end{array}\right]$ is causal and causally invertible, $L_{11}^{-1}(z)$ is causal, and $L_{12}(z)$ is strictly causal. If this holds, then all possible $H^{\infty}$ estimators of level $\gamma$ are given by

$$
F(z)=\left(L_{22}(z) S(z)-L_{21}(z)\right)\left(L_{11}(z)-L_{12}(z) S(z)\right)^{-1},
$$

where $S(z)$ is causal and such that $S\left(e^{j \omega}\right) S^{*}\left(e^{j \omega}\right)<I(S(z)$ is, in general, highly non-unique transfer matrix). An important choice results from taking $S(z)=0$, so that

$$
F_{c e n}(z)=-L_{21}(z) L_{11}^{-1}(z),
$$

which is the so-called "central" filter.

The proof of Theorem 2 can be found in [13]. The important implication of the Theorem 2 is that the solution to the $H^{\infty}$ estimation problem is highly nonunique. This is due to the fact that the suboptimal $H^{\infty}$ problem is expressed as a feasibility problem, rather than an optimization problem. We should mention that the aformentioned MATLAB implementation tools yield the central solution of Theorem 2.

\subsection{A Special Case: Paraunitary Filter Banks}

We notice that for the special case of a paraunitary analysis matrix $H(z)$, i.e., when $H(z) H^{*}\left(z^{-*}\right)=H^{*}\left(z^{-*}\right) H(z)=I$, we have from (7)

$$
\gamma_{s}^{2}=\frac{\sigma^{2}}{1+\sigma^{2}}
$$


and

$$
F_{s}(z)=\frac{1}{1+\sigma^{2}} L(z) H^{*}\left(z^{-*}\right)
$$

As we noted earlier, explicit closed-form expressions for $\gamma_{\text {opt }}$, the minimum achievable disturbance attenuation, are not always available when the causal constraint on the synthesis filters is imposed. Nonetheless, for paraunitary matrices $H(z)$, an expression for $\gamma_{\text {opt }}$ can be obtained. To this end, consider the $H^{\infty}$ suboptimal problem of solving for a causal estimator $F(z)$ which achieves

$$
\|[L(z)-F(z) H(z)-\sigma F(z)]\|_{\infty}<\gamma
$$

Assuming a paraunitary $H(z),(10)$ implies

$$
[L(z)-F(z) H(z)][L(z)-F(z) H(z)]^{*}+\sigma^{2} F(z) F^{*}\left(z^{-*}\right)<\gamma^{2}
$$

or, equivalently,

$$
[L(z)-F(z) H(z)] H^{*}\left(z^{-*}\right) H(z)[L(z)-F(z) H(z)]^{*}+\sigma^{2} F(z) F^{*}\left(z^{-*}\right)<\gamma^{2} .
$$

and, after obvious simplyfication,

$$
\left[L(z) H^{*}\left(z^{-*}\right)-F(z)\right]\left[L(z) H^{*}\left(z^{-*}\right)-F(z)\right]^{*}+\sigma^{2} F(z) F^{*}\left(z^{-*}\right)<\gamma^{2} .
$$

Since $L(z) H^{*}\left(z^{-*}\right) H(z) L^{*}\left(z^{-*}\right)=I$, we have

$$
\begin{aligned}
& {\left[L(z) H^{*}\left(z^{-*}\right)-F(z)\right]\left[L(z) H^{*}\left(z^{-*}\right)-F(z)\right]^{*}-\frac{\sigma^{2}}{1+\sigma^{2}} L(z) H^{*}\left(z^{-*}\right) H(z) L^{*}\left(z^{-*}\right)} \\
& +\sigma^{2} F(z) F^{*}\left(z^{-*}\right)+\frac{\sigma^{2}}{1+\sigma^{2}}<\gamma^{2}
\end{aligned}
$$

which, upon factorization, becomes

$$
\left[\frac{L(z) H^{*}\left(z^{-*}\right)}{\sqrt{1+\sigma^{2}}}-\sqrt{1+\sigma^{2}} F(z)\right]\left[\frac{L(z) H^{*}\left(z^{-*}\right)}{\sqrt{1+\sigma^{2}}}-\sqrt{1+\sigma^{2}} F(z)\right]^{*}+\frac{\sigma^{2}}{1+\sigma^{2}}<\gamma^{2} .
$$

Introducing $\left(\gamma^{\prime}\right)^{2}=\gamma^{2}-\frac{\sigma^{2}}{1+\sigma^{2}}$, the last inequality implies

$$
\left\|\frac{1}{\sqrt{1+\sigma^{2}}} L(z) H^{*}\left(z^{-*}\right)-\sqrt{1+\sigma^{2}} F(z)\right\|_{\infty}<\gamma^{\prime}
$$


Let us assume $H(z)=\sum_{i=-\infty}^{\infty} h_{i} z^{-i}$ and denote

$$
T(z)=\frac{1}{\sqrt{1+\sigma^{2}}} L(z) H^{*}\left(z^{-*}\right) .
$$

Our goal is to find a causal $F(z)$ which minimizes (24). Define the Hankel operator associated with $T(z)=\sum_{-\infty}^{\infty} t_{i} z^{-i}$ as

$$
\mathcal{H}_{T}=\left[\begin{array}{lll}
t_{-1} & t_{-2} & t_{-3} \\
t_{-2} & t_{-3} & \cdots \\
t_{-3} & \vdots & \\
\vdots & &
\end{array}\right]=\left[\begin{array}{llll}
h_{d+1}^{*} & h_{d+2}^{*} & h_{d+3}^{*} & \cdots \\
h_{d+2}^{*} & h_{d+3}^{*} & \cdots \\
h_{d+3}^{*} & \vdots \\
\vdots & &
\end{array}\right]
$$

By Nehari's theorem ([14]),

$$
\inf _{\text {causalF }(z)}\left\|\left[T(z)-\sqrt{1+\sigma^{2}} F(z)\right]\right\|_{\infty}=\bar{\sigma}\left(\mathcal{H}_{T}\right)
$$

Hence the achievable $\gamma$-level in (6) is given by

$$
\gamma_{\text {opt }}^{2}=\frac{\sigma^{2}}{1+\sigma^{2}}+\bar{\sigma}^{2}\left(\mathcal{H}_{T}\right)
$$

Clearly, when in addition $H(z)$ is FIR, if the delay $d$ is greater than the length of $H(z)$, the system $T(z)$ is going to be causal so that the Hankel operator is zero. In that case,

$$
\gamma_{o p t}^{2}=\frac{\sigma^{2}}{1+\sigma^{2}}
$$

and the optimal solution is given by:

$$
F(z)=\frac{1}{1+\sigma^{2}} L(z) H^{*}\left(z^{-*}\right) .
$$

When $H(z) H^{*}\left(z^{-*}\right)=I$, this is same as Wiener smoother solution of (8). Hence, when $H(z)$ is paraunitary and FIR, a delay equal to the length of $H(z)$ suffices to obtain the same performance as the non-causal solution.

For IIR filters, or for delays less than the length of the FIR $H(z),(13)$ offers a way of relating the achievable $\gamma$ performance of the filter bank system to the 
delay $d$. We should remark that computation of the Hankel norm of a transfer matrix is straightforward and simply requires computing the maximum singular value of a $n \times n$ matrix, where $n$ is the transfer matrix' McMillan degree.

We can summarize the previous discussion with the following lemma:

Lemma 1 The achievable $\gamma$-level for a signal reconstruction in a noisy paraunitary filter bank is given by

$$
\gamma_{o p t}^{2}=\frac{\sigma^{2}}{1+\sigma^{2}}+\bar{\sigma}^{2}\left(\mathcal{H}_{T}\right)
$$

where $\mathcal{H}_{T}$ is the Hankel operator associated with $T(z)=\frac{1}{\sqrt{1+\sigma^{2}}} L(z) H^{*}\left(z^{-*}\right)$, $\sigma^{2}$ is the noise variance and $\bar{\sigma}(\cdot)$ denotes the maximum singular value of its argument.

\subsection{The General Case: Minimum Delay}

It is of interest to obtain those values of delay $m$ that ensures that the performance of the estimator exceeds that of doing no estimation. In other words: of determining the value of delay that ensures $\gamma_{\text {opt }}<1$ (since $F(z)=0$ results in $\gamma=1$ ). For a single block case, the authors in [7] report examples with the minimum delay $m$ needed to obtain values of $\gamma_{o p t}<1$. This "worst-case non-estimability" has been studied in [15]-[16], from which it follows that that choosing $m$ greater than the number of non-minimum phase zeros of $H(z)$ is a sufficient condition for achieving $\gamma_{o p t}<1$. [In all the numerical examples performed, it was observed that this condition is also necessary.]

Finally, once $\gamma_{\text {opt }}$ has been obtained, the actual filters $F(z)$ can be found via standard $H^{\infty}$ techniques. These techniques are based on a solution of a Riccati equation, leading to the J-spectral factorization in state-space form. The corresponding central solution is, in general, an IIR filter of the same McMillan degree as $\left[\begin{array}{lll}H(z) & L(z)\end{array}\right]^{T}$, which could be rather high.

In practice, however, IIR synthesis filters are rarely used in filter bank applications. (One major reason is that finite-precision implementations may lead to limit cycles, or other forms of numerical instability.) Therefore in the remainder of this paper we shall focus on FIR synthesis filters. This has one further advantage: the $H^{\infty}$ design procedure can be reduced to a finite (rather than infinite) dimensional semi-definite program, so that it is possible to effectively optimize the filter weights over criteria in addition to the $H^{\infty}$ constraint. 


\section{FIR Synthesis Filters: State Space Formulation}

In this section we shall derive the state-space representation of the transfer function $T_{F}(z)$ for FIR synthesis filters. Given this state-space representation, we shall pose the $H^{\infty}$ optimization problem as a semi-definite programming problem, which allows for efficient numerical solutions.

To obtain the state-space description of $T_{F}(z)$, we first need to introduce the state-space descriptions for $H(z), F(z)$, and $L(z)$.

The state space description of the polyphase analysis filters $H(z)$, with noisy subchannels, is given by

$$
\begin{gathered}
\beta_{i+1}=\underbrace{A_{h}}_{A_{H}} \beta_{i}+\underbrace{\left[\begin{array}{ll}
B_{h} & 0
\end{array}\right]}_{B_{H}}\left[\begin{array}{c}
\mathbf{u}_{i} \\
\mathbf{v}_{i}
\end{array}\right] \\
\mathbf{y}_{i}=\underbrace{C_{h}}_{C_{H}} \beta_{i}+\underbrace{\left[\begin{array}{ll}
D_{h} & I
\end{array}\right]}_{D_{H}}\left[\begin{array}{c}
\mathbf{u}_{i} \\
\mathbf{v}_{i}
\end{array}\right]
\end{gathered}
$$

where $\left(A_{h}, B_{h}, C_{h}, D_{h}\right)$ are the matrices in the state space realization of the transfer function $H(z), \mathbf{u}_{i}$ is the input sequence, and $\mathbf{v}_{i}$ is the noise sequence.

The state space representation of the delay $L(z)=z^{-d} I$ is given as

$$
\begin{aligned}
& \eta_{i+1}=\underbrace{\left[\begin{array}{cc}
0_{2 \times(2 d-2)} & 0_{2 \times 2} \\
I_{(2 d-2) \times(2 d-2)} & 0_{(2 d-2) \times 2}
\end{array}\right]}_{A_{L}} \eta_{i}+\underbrace{\left[\begin{array}{cc}
I_{2 \times 2} & 0_{2 \times 2} \\
0_{(2 d-2) \times 2} & 0_{(2 d-2) \times 2}
\end{array}\right]}_{B_{L}}\left[\begin{array}{l}
\mathbf{u}_{i} \\
\mathbf{v}_{i}
\end{array}\right] \\
& \mathbf{u}_{i-d}=\underbrace{\left[\begin{array}{ll}
0 & I
\end{array}\right]}_{C_{L}} \eta_{i}
\end{aligned}
$$

We assume FIR synthesis filters, i.e.,

$$
F(z)=F_{0}+F_{1} z^{-1}+F_{2} z^{-2}+\ldots+F_{L-1} z^{-(L-1)} .
$$

The state space structure of $F(z)$ is of the form

$$
\xi_{i+1}=\underbrace{\left[\begin{array}{cc}
0_{(2 d-2) \times 2} & 0_{2 \times 2} \\
I_{2 \times 2} & 0_{2 \times(2 d-2)}
\end{array}\right]}_{A_{F}} \xi_{i}+\underbrace{\left[\begin{array}{c}
I_{2 \times 2} \\
0_{(2 d-2) \times 2}
\end{array}\right]}_{B_{F}} \mathbf{y}_{i}
$$




$$
\hat{\mathbf{u}}_{i-d}=\underbrace{\left[F_{1} \ldots F_{L-1}\right]}_{C_{F}} \xi_{i}+\underbrace{F_{0}}_{D_{F}} \mathbf{y}_{i}
$$

To obtain the state-space structure for $T_{F}(z)$, we group all the state variables into a single state vector

$$
\mathbf{x}_{i}=\left[\begin{array}{lll}
\beta_{i} & \eta_{i} & \xi_{i}
\end{array}\right]^{T}
$$

Let us denote

$$
\begin{gathered}
A_{T}=\left[\begin{array}{ccc}
A_{H} & 0 & 0 \\
0 & A_{L} & 0 \\
B_{F} C_{H} & 0 & A_{F}
\end{array}\right], \quad B_{T}=\left[\begin{array}{c}
B_{H} \\
B_{L} \\
B_{F} D_{H}
\end{array}\right], \\
C_{T}=\left[\begin{array}{lll}
-F_{0} C_{H} & C_{L} & -C_{F}
\end{array}\right], \quad D_{T}=-F_{0} D_{H} .
\end{gathered}
$$

It can be shown that the state space equations for $T_{F}(z)$ then can be written as

$$
\begin{gathered}
\mathbf{x}_{i+1}=A_{T} \mathbf{x}_{i}+B_{T}\left[\begin{array}{l}
\mathbf{u}_{i} \\
\mathbf{v}_{i}
\end{array}\right] \\
\mathbf{y}_{i}=C_{T} \mathbf{x}_{i}+D_{T}\left[\begin{array}{l}
\mathbf{u}_{i} \\
\mathbf{v}_{i}
\end{array}\right]
\end{gathered}
$$

We now invoke a standard representation of the $H_{\infty}$ norm as a convex constraint parametrized over the matrices obtained from the state-space representation. This can be stated as follows:

Lemma 2 (Bounded Real) Given any transfer function $T_{F}(z)=D_{T}+$ $C_{T}\left(z I-A_{T}\right)^{-1} B_{T}$, we have

$$
\left\|T_{F}\right\|_{\infty}<\gamma
$$


if and only if the following LMI in $X$ is feasible:

$$
\begin{aligned}
& {\left[\begin{array}{ccc}
A_{T}^{T} X A_{T} & A_{T}^{T} X B_{T} & C_{T}^{T} \\
B_{T}^{T} X A_{T} & B_{T}^{T} X B_{T}-\gamma I & D_{T}^{T} \\
C_{T} & D_{T} & -\gamma I
\end{array}\right]<0} \\
& X>0
\end{aligned}
$$

Proof: The proof can be found in several places (see, e.g., [23], [24]).

Notice that for a given synthesis filter $H(z)$, and a given delay $d$, once we choose the order of $F(z)$, the matrices $A_{T}$ and $B_{T}$ are fixed. Thus, (16) is an LMI (linear matrix inequality) in $X, C_{T}$ and $D_{T}$ (and hence in $X$ and the filter weights of $F(z)$, since these appear linearly in $C_{T}$ and $D_{T}$ ), and we can re-state the suboptimal $H^{\infty}$ problem as the following semi-definite program (SDP):

Problem 3 (SDP formulation of the $H^{\infty}$ reconstruction problem) Given matrices $A_{T}$ and $B_{T}$ in the state-space realization of $T_{F}(z)$, solve

$$
\min _{X, C_{T}, D_{T}} \gamma
$$

subject to (16).

This SDP can be solved using efficient algorithms such as the primal-dual method ([18], [19]).

\section{Mixed $H^{2} / H^{\infty}$ Signal Reconstruction}

As we discussed in Section 3 and at the end of the Section 4, the solution to the $H^{\infty}$ estimation problem is highly non-unique. One way to remove this non-uniqueness is to optimize some other criterion besides the $H^{\infty}$ feasibility constraint. A natural choice for a criterion in a filter bank reconstruction context is to minimize the $H^{2}$ norm of the transfer matrix $T_{F}(z)$, i.e., $\left\|T_{F}\right\|_{2}$. We notice that when $T_{F}$ is a matrix, then

$$
\left\|T_{F}\right\|_{2}=\operatorname{trace}\left(T_{F} T_{F}^{*}\right)^{\frac{1}{2}} .
$$


Thus the goal of unconstrained $H^{2}$ optimization in the finite horizon case is to minimize the Frobenius norm of the matrix $T_{F}$. In the infinite-horizon case, we have

$$
\left\|T_{F}\right\|_{2}=\left(\frac{1}{2 \pi} \int_{0}^{2 \pi} \operatorname{trace}\left[T_{F}\left(e^{j \omega}\right) T_{F}^{*}\left(e^{j \omega}\right)\right] d \omega\right)^{\frac{1}{2}}
$$

By introducing an $H^{\infty}$ constraint to the $H^{2}$ optimization problem, we can exploit the non-uniqueness of the solution to the $H^{\infty}$ problem in order to improve some other performance aspect of the estimator besides its obvious robustness. This leads to the mixed $H^{2} / H^{\infty}$ criterion (see, e.g., [20], [22], [21]). and results in the estimator with the best average performance among all estimators achieving the same optimal $\gamma$-level. Thus, the mixed estimator yields a smaller $H^{2}$ norm than the one obtained from the aformentioned central solution to the $H^{\infty}$ problem. We should also point out that the mixed estimator achieves the optimal $H^{2}$ performance whenever the optimal $H^{2}$ estimator satisfies the $H^{\infty}$ bound.

Problem 4 (Mixed $H^{2} / H^{\infty}$ Signal Reconstruction) Given $\gamma>0$, find a causal polyphase synthesis filter $F(z)$ that minimizes the $H^{2}$ norm of the transfer function $T_{F}(z)=[L(z)-F(z) H(z) \quad-\sigma F(z)]$, subject to the $H^{\infty}$ norm of $T_{F}(z)$ being less than $\gamma$. In other words, find a causal $F(z)$ that satisfies

$$
\min _{F(z)}\left\|T_{F}(z)\right\|_{2}
$$

$$
\text { subject to }\left\|T_{F}(z)\right\|_{\infty} \leq \gamma
$$

Note that, in the frequency domain, both the objective and the constraints are convex. While it is possible to solve the above problem by sampling in the frequency domain, this generally leads to an infinite-dimensional SDP, since we will have an infinite number of constraints (one for each frequency).

Therefore, as in the pure $H^{\infty}$ problem, in order to obtain a finite-dimensional SDP, we seek a way to restate the problem of finding the optimal $H^{2} / H^{\infty}$ solution in terms of its state-space representation. To this end, assuming the state-space description of the transfer function $T_{F}(z)$ in (15), we first state a result on the representation of the $H^{2}$ norm as an LMI constraint in the following lemma.

Lemma 3 ( $H^{2}$ Norm bound) Given any transfer function $T_{F}(z)=D_{T}+$ 
$C_{T}\left(z I-A_{T}\right)^{-1} B_{T}$, it holds that

$$
\left\|T_{F}\right\|_{2}^{2}<\alpha^{2}
$$

if and only if the following LMI in $Y$ and $S$ is feasible:

$$
\begin{aligned}
& {\left[\begin{array}{cc}
A_{T}^{T} Y A_{T}-Y & A_{T}^{T} Y B_{T} \\
B_{T}^{T} Y A_{T} & B_{T}^{T} Y B_{T}-I
\end{array}\right]<0} \\
& {\left[\begin{array}{ccc}
Y & 0 & C_{T}^{T} \\
0 & I & D_{T}^{T} \\
C_{T} & D_{T} & S
\end{array}\right]>0} \\
& \operatorname{Tr}(S)-\alpha^{2}<0 \\
& Y>0
\end{aligned}
$$

Proof: The proof can be found in [25], [26].

We now use results of Lemma 2 and Lemma 3 to formulate the mixed $H^{2} / H^{\infty}$ optimization problem as a SDP in state-space.

Problem 5 (SDP Formulation of the Mixed $H^{2} / H^{\infty}$ Problem) The mixed $H^{2} / H^{\infty}$ signal reconstruction problem (17) is equivalent to the following SDP:

$$
\min _{C_{T}, D_{T}} \alpha^{2}
$$

subject to (16), (18)

Notice that $\gamma$ in (16) must be feasible, i.e., we must have

$$
\gamma \geq\left\|T_{F}\right\|_{\infty}=\gamma_{o p t} .
$$

Moreover, as in the SDP formulation of the pure $H^{\infty}$ optimization problem, for a given delay and a given analysis filter length, the matrices $A_{T}$ and $B_{T}$ are fixed and both (16) and (18) are LMIs in $X, Y, S, \alpha^{2}$, and $C_{T}$ and $D_{T}$ (and hence in the filter weights of $F(z)$, since $C_{T}$ and $D_{T}$ are linear in these weights). 


\section{Risk Sensitive Signal Reconstruction}

In Section 5, we showed how to remove inherent non-uniqueness of the $H^{\infty}$ signal reconstruction problem by additionally optimizing $H^{2}$ norm of the transfer matrix $T_{F}(z)$, which led to the mixed $H^{2} / H^{\infty}$ signal reconstruction. In this section, we show how to remove the non-uniqueness by searching for $H^{\infty}$ optimal synthesis filters with minimum risk sensitive cost, yielding the minimum entropy synthesis filters [13]. These filters minimize exponential-quadratic criterion

$$
\operatorname{Eexp}\left(\frac{1}{2 \gamma^{2}}\|e\|^{2}\right)
$$

for some $\gamma^{2}$. Minimizing (19) is equivalent to minimizing

$$
\gamma^{2} \log \left[\operatorname{Eexp}\left(\frac{1}{2 \gamma^{2}}\|e\|^{2}\right)\right]
$$

The criterion thus penalizes occasional occurrence of large values of reconstruction error more than frequent occurrence of small to moderate reconstruction errors.

The design of risk-sensitive FIR synthesis filters can be posed as a finite dimensional convex optimization problem and thus efficiently solved using interior point methods [18]. To show this, we first use state space description introduced in Section 4 to give an alternative statement of the $H^{\infty}$ feasibility condition. To this end, we proceed with stating Kalman-Yakubovich-Popov (KYP) lemma:

Lemma 4 (KYP Lemma) Consider the observable pair $(A, C)$. Then the following two statements are equivalent

(1) $S_{y}(z) \geq 0$ for all $z=e^{j \omega} \notin \lambda(F)$ where

$$
S_{y}(z)=\left[C(z I-A)^{-1} I\right]\left[\begin{array}{cc}
Q & S \\
S^{*} & R
\end{array}\right]\left[\begin{array}{c}
\left(z^{-1} I-A^{*}\right)^{-1} C^{*} \\
I
\end{array}\right]
$$

(2) There exists a Hermitian $Z$ such that

$$
\left[\begin{array}{cc}
-Z+A Z A^{*} & S+A Z C^{*} \\
S^{*}+C Z A^{*} & R+C Z C^{*}
\end{array}\right] \geq 0
$$


The proof of Lemma 4 can be found in [13]. Assume state-space description of the error transfer matrix $T_{F}(z)$ as in (15). Using Schur complement, it can easily be shown that condition $\left\|T_{F}\right\|_{\infty}<\gamma$ is equivalent to

$$
\left[\begin{array}{cc}
I & T_{F}\left(e^{j \omega}\right) \\
T_{F}^{*}\left(e^{j \omega}\right) & \gamma^{2} I
\end{array}\right] \geq 0, \quad 0 \leq \omega \leq 2 \pi
$$

Further, it can be shown (see, e.g., [15]) that

$$
\left[\begin{array}{cc}
I & T_{F}\left(e^{j \omega}\right) \\
T_{F}^{*}\left(e^{j \omega}\right) & \gamma^{2} I
\end{array}\right]=\left[C\left(e^{j \omega} I-A\right)^{-1} I\right]\left[\begin{array}{cc}
0 & S \\
S^{*} & R
\end{array}\right]\left[\begin{array}{c}
\left(e^{-j \omega} I-A^{*}\right)^{-1} C^{*} \\
I
\end{array}\right]
$$

where

$$
\begin{gathered}
A=A_{T}^{*}, \quad S=\left[\begin{array}{cc}
C_{T}^{*} & 0
\end{array}\right], \\
C=\left[\begin{array}{c}
0 \\
B_{T}^{*}
\end{array}\right], R=\left[\begin{array}{cc}
I & D_{T} \\
D_{T}^{*} & \gamma^{2} I
\end{array}\right] .
\end{gathered}
$$

Therefore, from KYP Lemma we conclude that the set of $\gamma$-level $H^{\infty}$ optimal FIR filters is a convex set $\mathcal{F}_{\gamma}$ of all $F(z)$ for which there exists a hermitian $Z$ such that condition (21) is satisfied, with $(A, C, R, S)$ defined as in (22).

For the infinite horizon case, the risk sensitive synthesis filters design can be formulated as

$$
\min _{F \in \mathcal{F}_{\gamma}}-\int_{-\pi}^{\pi} \log \left(\operatorname{det}\left(\gamma^{2}-T_{F}\left(e^{j \omega}\right) T_{F}^{*}\left(e^{j \omega}\right)\right)\right) d \omega
$$

The cost function in (23) is a convex function of the coefficients of the synthesis filters. Moreover, since $\mathcal{K}_{\gamma}$ is a convex set, the minimization in (23) is a convex optimization problem. It is possible to convert the cost function from (23) into a more compact expression involving previously introduced state space description as in [15]. This leads to the so-called "constrained analytical centering" problem:

$$
\begin{gathered}
\operatorname{minimize}-\log \left(\operatorname{det}\left(R+C Z C^{*}\right)\right) \\
\text { subject to }(21) .
\end{gathered}
$$


Notice that the optimization problem in (24) has a nonlinear convex barrier function as the cost function and a convex linear matrix inequality (LMI) constraint. It can thus be efficiently solved using interior point methods.

\section{Simulation Results and Discussion}

In this section, we illustrate the performance of the $H^{\infty}$ optimal FIR synthesis filters given IIR analysis filters in a 2-band filter bank. As the $H^{\infty}$ approach does not put any constraints on the choice of the analysis filters, we may choose them arbitrarily. For simplicity, the fifth order Butterworth filters shown in Figure 4 were chosen for the analysis filters. Furthermore, we compare the average performance of the mixed $H^{2} / H^{\infty}$ optimal reconstruction filters with the central $H^{\infty}$ solution as obtained in [7]. ${ }^{\dagger}$ [For this particular example, the risk sensitive and the mixed $H^{2} / H^{\infty}$ optimal synthesis filters coincide.]

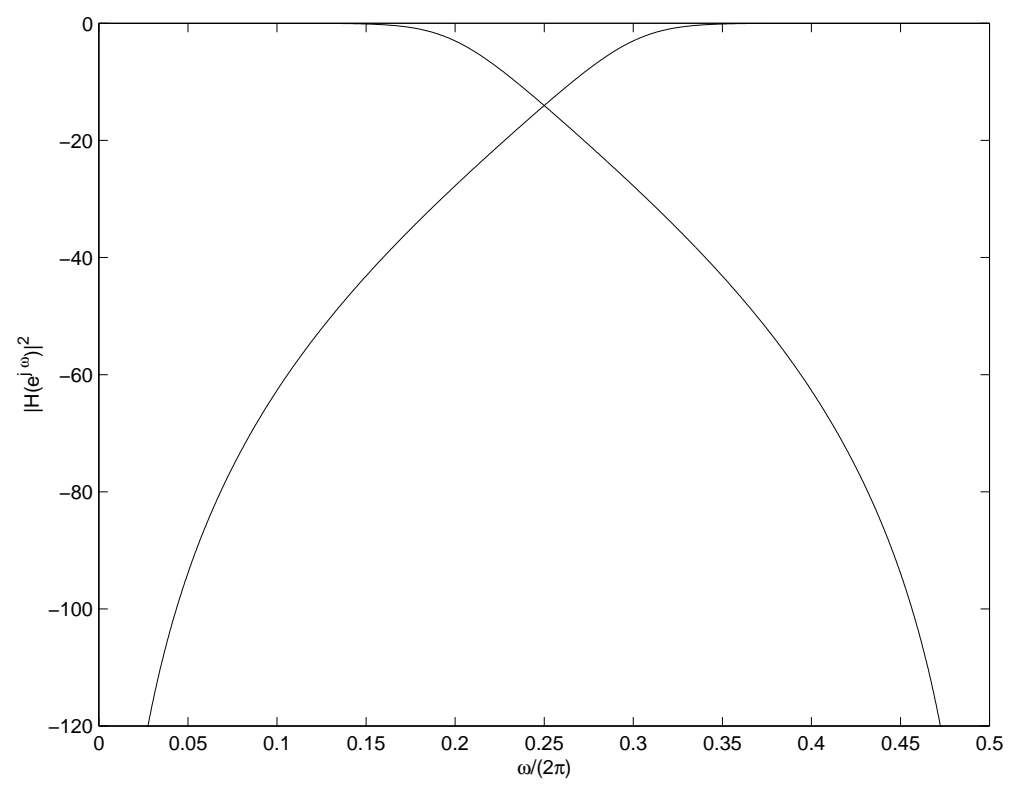

Fig. 4. Frequency response of analysis filters

We have chosen the length of the FIR synthesis filters to be $L=16$. The frequency responses of the corresponding mixed $H^{2} / H^{\infty}$ optimal synthesis filters are shown in Figure 5.

Figure 6 shows the largest singular value of the error transfer function, $T_{F}\left(e^{j \omega}\right)$ as a function of frequency. Although the area under this curve is not, strictly

$\dagger$ In general, the central $H^{\infty}$ solution is an IIR filter and in the following simulations we approximate this IIR filter by an FIR filter obtained by truncation to a length equal to the length of the mixed $H^{2} / H^{\infty}$ solution. 


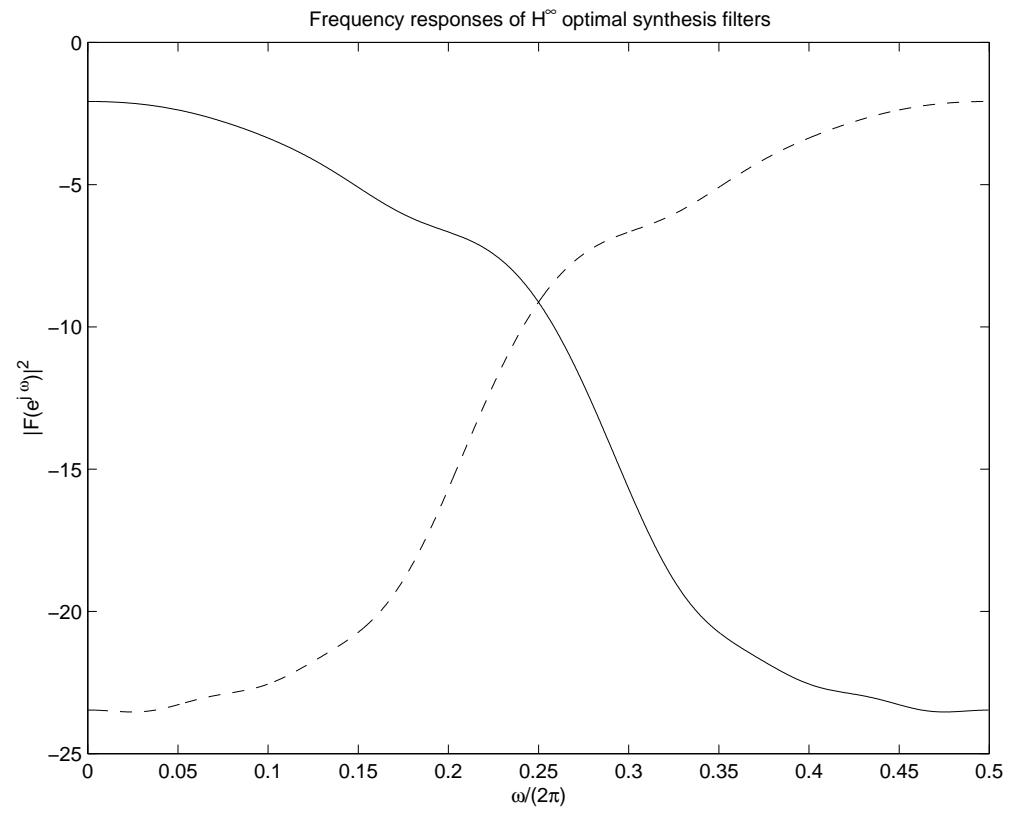

Fig. 5. Frequency response of the mixed $H^{2} / H^{\infty}$ optimal synthesis filters, $L=16$, $d=5, \gamma=\gamma_{o p t}$

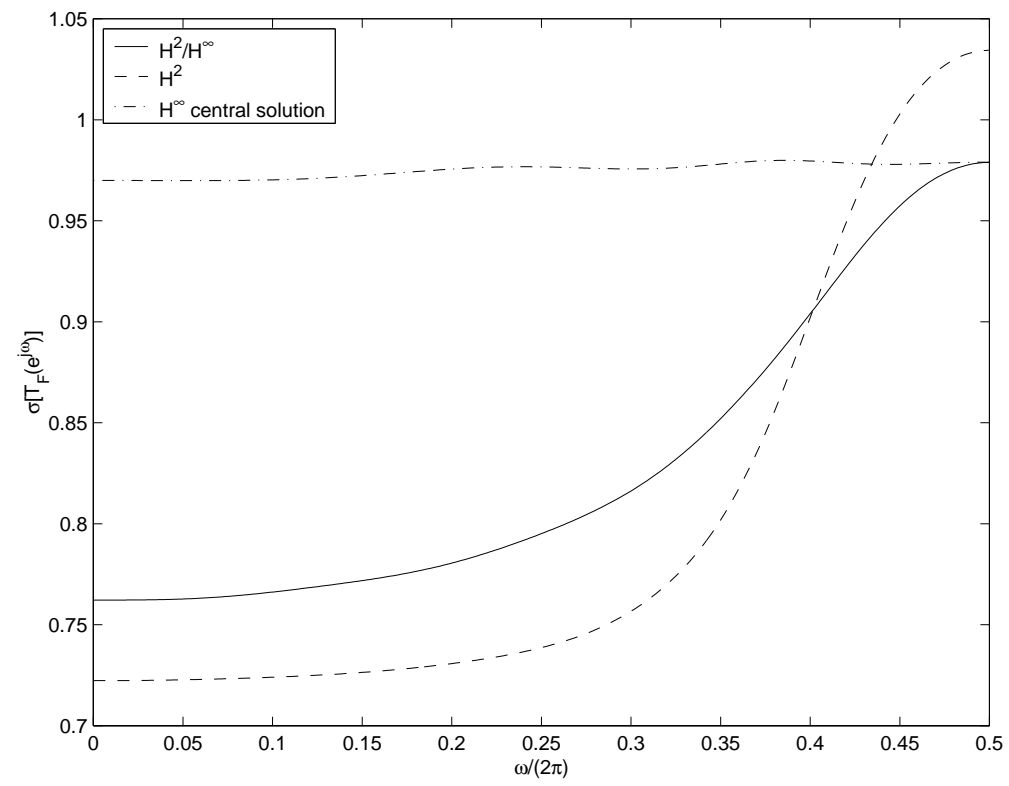

Fig. 6. $\bar{\sigma}\left[T_{F}\left(e^{j \omega}\right)\right]$ vs. $\omega, \gamma=\gamma_{o p t}$

speaking, the $H^{2}$ norm of $T_{F}\left(e^{j \omega}\right)$ (since we also need to add the contribution from the second singular value), it is somewhat indicative of the $H^{2}$ norm, and hence the average performance of the filters. Figure 6 clearly shows that the $H^{2}$-optimal synthesis filter has the smallest area under the curve, which is a result of the fact that, under given stochastic assumptions, the $H^{2}$-optimal synthesis filter have the best average performance among all possible causal synthesis filters. On the other hand, the $H^{\infty}$-optimal synthesis filters yield the 
error spectra with the smallest peak. Thus the $H^{\infty}$-optimal synthesis filters (both central and mixed solution) guarantee the best worst-case performance among all causal estimators.

It is also clear from Figure 6 that the $H^{2}$-optimal synthesis filter can have poor performance if the disturbance signals have high frequency components, since the value of the error spectrum at these frequencies is large. This is indicative of the fact that $H^{2}$-optimal filters may have poor robustness properties. The $H^{\infty}$ filters, on the other hand, have inferior average performance. The central $H^{\infty}$-optimal solution, in particular, has poor average performance, since the curve of $\bar{\sigma}\left[T_{F}\left(e^{j \omega}\right)\right]$ is quite flat. The mixed $H^{2} / H^{\infty}$ solution, however, by virtue of its very construction, has an area under the $\bar{\sigma}\left[T_{F}\left(e^{j \omega}\right)\right]$ curve that is comparable to that of the $H^{2}$-optimal solution. Thus it achieves close to optimal average performance, while being robust at the same time.

To compare the performance of the various filters for the signal reconstruction application, we adopt the SNR of the input signal to the reconstruction error $([6])$

$$
S N R_{r}=10 \log _{10}\left(\frac{\sum_{k} u^{2}(k)}{\sum_{k}(u(k-m)-\hat{u}(k))^{2}}\right) .
$$

Figure 7 compares the average performances (in terms of the above reconstruction SNR as a function of the delay, $m$ ) for the optimal mixed $H^{2} / H^{\infty}, H^{2}$, and central $H^{\infty}$ solution. The input signal and noise are modeled as white sequences yielding subband signal-to-noise ratios of $0 \mathrm{~dB}$. Thus $S N R_{r}$ measures the improvement with respect to $0 \mathrm{~dB}$ obtained from performing reconstruction. As can be seen from Figure 7 , the mixed $H^{2} / H^{\infty}$ optimal reconstruction filters are on average slightly outperformed by the $H^{2}$ optimal reconstruction filters. The average performance of the filters obtained from the central $H^{\infty}$ solution, however, is significantly worse than that of either the $H^{2}$ or mixed $H^{2} / H^{\infty}$ one. As mentioned earlier, this is clearly expected from Figure 6 .

Finally, Figure 8 compares the worst-case performances (again in terms of the reconstruction SNR as a function of the delay, $m$ ) for the optimal mixed $H^{2} / H^{\infty}, H^{2}$, and central $H^{\infty}$ solution. Here, strictly speaking, the performances are not really "worst-case", but rather those that are obtained from input and noise signals that have most of their frequency components at frequencies where $\bar{\sigma}\left[T_{F}\left(e^{j \omega}\right)\right]$ is large - it is therefore only indicative of the worstcase performance. As can be seen, the central $H^{\infty}$-optimal and mixed $H^{2} / H^{\infty}$ optimal filters significantly outperform the $H^{2}$-optimal filter, in terms of the worst-case performance. ${ }^{\ddagger}$

$\ddagger$ We should mention, in passing, that for large values of delay, $m$, the average and worst-case performances of the $H^{2}$ - and $H^{\infty}$-optimal filters are close, since it is well 


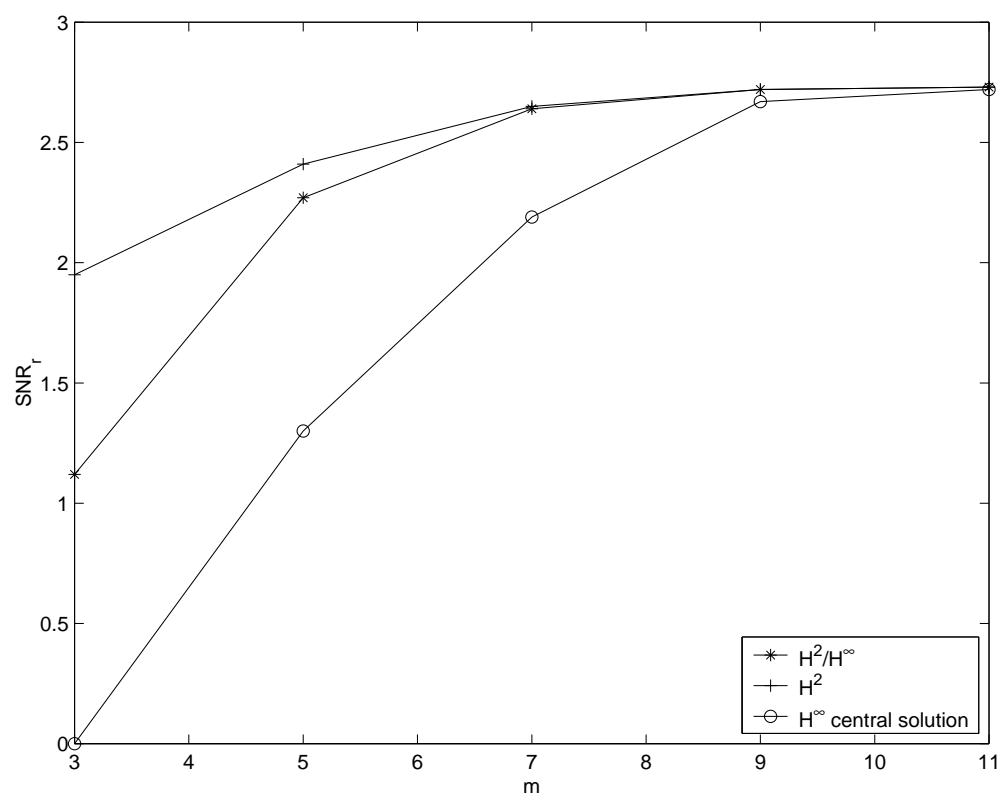

Fig. 7. $S N R_{r}$ vs. $m$ with white noise input disturbance

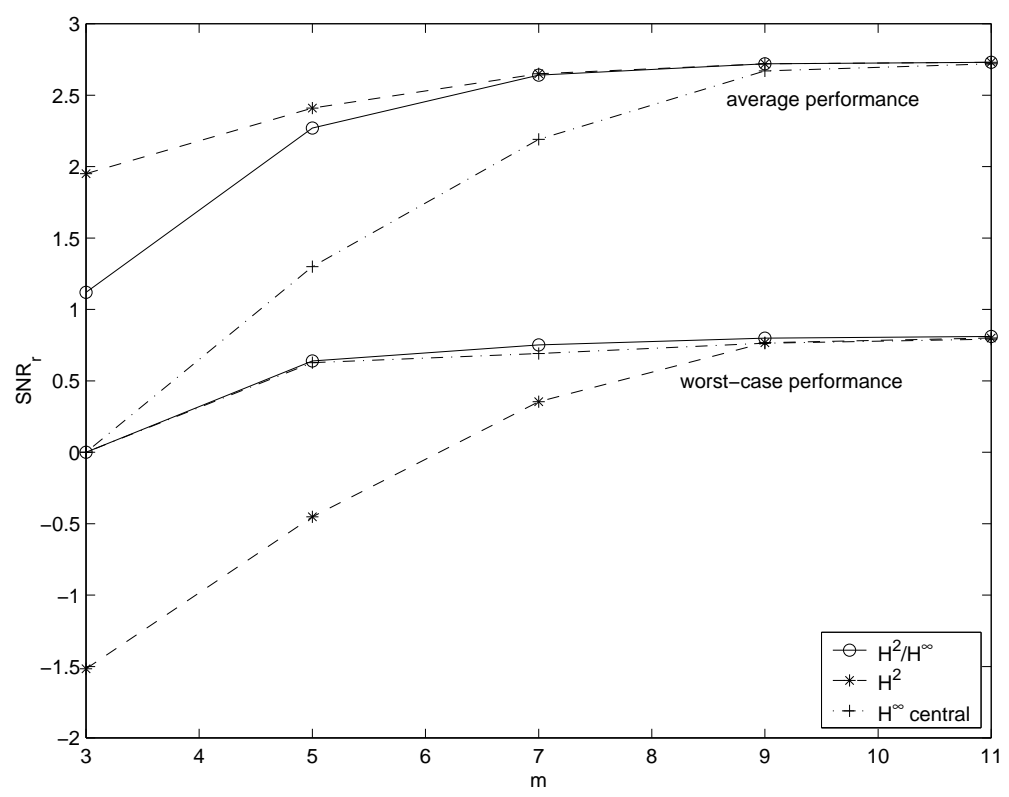

Fig. 8. $S N R_{r}$ vs. $m$ for worst-case input and noise signals.

In summary, the mixed $H^{2} / H^{\infty}$-optimal filter appears to achieve the best of both worlds: it has average performance comparable to that of the $H^{2}$-optimal solution, while it significantly outperforms this filter in terms of the worst-case performance.

known that the noncausal $H^{2}$ - and $H^{\infty}$-optimal solutions coincide. 


\section{Summary}

The design of multirate filter banks often assumes that the subbands of the filter bank are noise free. However, quantization and encoding cause the corruption of the signal in the subbands and may thus significantly deteriorate the reconstructed signal. Moreover, the statistical properties of such mechanisms are often hard to determine so that statistical methods (such as the Kalman filter) for reconstructing the corrupted signal are not always applicable.

In this paper, we have attempted to address the signal reconstruction problem from an $H^{\infty}$ estimation point of view, which provides robustness against statistical uncertainty. We give an explicit solution for the case of paraunitary analysis filters. Exploiting the non-uniqueness of the $H^{\infty}$ solution, we design synthesis filters which both satisfy an $H^{\infty}$ bound and optimize for the average performance, yielding the so-called mixed $H^{2} / H^{\infty}$ optimal solution to the reconstruction problem. The general solution to the $H^{\infty}$-optimal signal reconstruction problem is an IIR synthesis filter, which may be of a high order and thus difficult to implement. To alleviate these implementation issues, we have further restricted the synthesis filters to be FIR. This enabled us to state the design problem as one of convex optimization (in particular, a semi-definite program) for which efficient numerical algorithms exist.

\section{References}

[1] P. P. Vaidyanathan, Multirate Systems and Filter Banks, Prentice-Hall, Englewood Cliffs, NJ, 1993.

[2] M. Vetterli and J. Kovacevic, Wavelets and Subband Coding, Prentice-Hall, Englewood Cliffs, NJ, 1995.

[3] G. Strang and T. Nguyen, Wavelets and Filter Banks, Wellesley-Cambridge Press, Wellesley, MA, 1997.

[4] R. A. Haddad and N. Uzun, "Modeling, analysis and compensation of quantization effects in M-band subband codecs," Proc. ICASSP '93, pp. III173-176.

[5] P. P. Vaidyanathan, "On coefficient-quantization and computational rounoff effects in lossless multirate filter banks," IEEE Trans. Signal Processing, vol. 39, no. 4, pp. 1006-1008, April 1991.

[6] B.-S. Chen, C.-W. Lin, and Y.-L. Chen, "Optimal signal reconstruction in noisy filter banks: multirate Kalman filtering approach," IEEE Transactions on Signal Processing, vol. 43, no. 11, November 1995. 
[7] T. Chen and B. A. Francis, "Design of multirate filter banks by $H^{\infty}$ optimization," IEEE Transactions on Signal Processing, vol. 43, no. 12, December 1995.

[8] R. N. Banavar and A. A. Kalele, "A Mixed Norm Performance Measure for the Design of Multirate Filterbanks," IEEE Trans. on Sig. Process., vol. 49, no. 2, February 2001.

[9] A. S. Mehr and T. Chen, "Design of Nonuniform Multirate Filter Banks by Semidefinite Programming," IEEE Trans. on Circuits and Systems-II, vol. 47, no. 11, November 2000.

[10] Y.-M. Cheng, B.-S. Chen, and L.-M. Chen, "Minimax deconvolution design of multirate systems with channel noises: a unified approach," IEEE Transactions on Signal Processing, vol. 47, no. 11, pp. 3145-3149, November 1999.

[11] B.-S. Chen, C.-L. Tsai, and Y.-F. Chen, "Mixed $H^{2} / H^{\infty}$ Filtering Design in Multirate Transmultiplexor Systems: LMI Approach," IEEE Trans. on Sig. Process., vol. 49, no. 11, pp. 2693-2701, November 2001.

[12] Y.-M. Cheng and B.-S. Chen, "Nonuniform filter bank design with noise," IEEE Transactions on Signal Processing, vol. 46, no. 9, September 1998.

[13] B. Hassibi, A. H. Sayed, and T. Kailath, Indefinite-Quadratic Estimation and Control: A Unified Approach to $H^{2}$ and $H^{\infty}$ Theories, SIAM 1998.

[14] Z. Nehari, "On bounded biliniear forms," Ann. Math., vol. 15, pp. 153-162, 1957.

[15] B. Hassibi, A. T. Erdogan, and T. Kailath, "Equalization with an $H^{\infty}$ Criterion," submitted to IEEE Transaction on Information Theory, 1998.

[16] B. Hassibi and T. Kailath, "On optimal solutions to two-block $H^{\infty}$ problems," submitted to IEEE Transaction on Automatic Control, 1998.

[17] U. Shaked and Y. Theodor, " $H^{\infty}$-optimal Estimation: A Tutorial," Proceedings of the IEEE Conference on Decision and Control, pp. 2278-2286, Tucson, AZ, 1992 .

[18] S. Boyd, L. El Ghaoui, E. Feron, and V. Balakrishnan, Linear Matrix Inequalities in System and Control Theory, SIAM Studies in Applied Mathematics, 1994.

[19] S.-P. Wu and S. Boyd, SDPSOL: A Parser/Solver for Semidefinite Programming and Determinant Maximization Problems with Matrix Structure. User's Guide, Beta Version. Stanford University, 1996.

[20] P. P. Khargonekar and M. A. Rotea, "Mixed $H^{2} / H^{\infty}$ filtering," Proc. IEEE Conference on Decision and Control, Tucson, AZ, pp. 2299-3004, December 1992.

[21] M. Sznaier, "An exact solution to general SISO mixed $H^{2} / H^{\infty}$ problems via convex optimization," IEEE Transactions on Automatic Control, vol. 39, no. 3, pp. 2511-2517. 
[22] K. Zhou, K. Glover, B. Bodenheimer, and J. C. Doyle, "Mixed $H^{2}$ and $H^{\infty}$ performance objectives I and II," IEEE Transactions on Automatic Control, vol. 39 , no. 8, pp. 1564-1587.

[23] K. Zhou, J. C. Doyle, and K. Glover, Robust and Optimal Control, Prentice Hall, Englewood Cliffs, NJ, 1996.

[24] M. Green and D. J. N. Limebeer, Linear Robust Control, Prentice Hall, Englewood Cliffs, NJ 1995.

[25] Robert E. Skelton, Tetsuya Iwasaki, and Karlos M. Grigoriadis, A Unified Algebraic Approach to Linear Control Design, Taylor and Francis, London, Bristol, PA, 1997.

[26] H. A. Hindi, B. Hassibi, S. P. Boyd, "Multiobjective $H^{2} / H^{\infty}$-Optimal Control via Finite Dimensional Q-Parametrization and Linear Matrix Inequalities," Proc. ACC, Philadelphia, PA, June 1998. 